\title{
Fluoride-containing mouthrinses in three European countries
}

\author{
Fusao Nishikawara*1,2, Yoshiaki Nomura*1,2, Yoh Tamaki*1,2,3, Seiko Katsumura*4, \\ Yoshinobu Asada*5, Nobuhiro Hanada*2 and Poul Erik Petersen*6 \\ *1 Department of Preventive Dentistry and Public Health, Tsurumi University School of Dental Medicine \\ 2-1-3 Tsurumi, Tsurumi-ku, Yokohama 230-8501, JAPAN \\ *2 Department of Oral Health, National Institute of Public Health \\ 2-3-6 Minami, Wako, Saitama 351-0197, JAPAN \\ *3 Division of Oral Health, Department of Health Science, Kanagawa Dental College \\ 82 Inaoka-cho, Yokosuka, Kanagawa 238-8580, JAPAN \\ ${ }^{* 4}$ Department of Anatomy, Tsurumi University School of Dental Medicine \\ 2-1-3 Tsurumi, Tsurumi-ku, Yokohama 230-8501, JAPAN \\ *5 Department of Pediatric Dentistry, Tsurumi University School of Dental Medicine \\ 2-1-3 Tsurumi, Tsurumi-ku, Yokohama 230-8501, JAPAN \\ *6 World Health Organization, Global Oral Health Programme, \\ Department of Chronic Disease and Health Promotion \\ 20 Avenue Appia, CH-1211 Geneva 27, SWITZERLAND
}

\begin{abstract}
The caries-preventive effect of fluoride is well known. The widespread use of fluoride-containing toothpastes and mouthrinses has been recommended to reduce dental caries. Many kinds of fluoride containing toothpastes are available in Japan. However, only two kinds of mouthrinses which contain fluoride are comically available. The aim of this study was to investigate the current status of fluoride-containing mouthrinses on the market in three European countries. Fluoride-containing mouthrinses were purchased from retail outlets in Athens, Amsterdam and Geneva. The labels of ingredients were studied to determine the type and concentration of fluoride. Most of the mouthrinses contained sodium fluoride $(90 \%)$; some contained stannous fluoride $(5 \%)$ or potassium fluoride $(7 \%)$. The concentrations of fluoride ranged from $100 \mathrm{ppm}$ to $500 \mathrm{ppm}$.
\end{abstract}

\section{Key words}

Fluoride,

Mouthrinses,

Prevention of dental caries
The effectiveness of fluoride for the prevention of dental caries has been clearly established. There are several methods for the application of the fluoride, including water fluoridation, varnishes, and several other commercially available professional and self-applied products. In Japan, according to the water quality standards of the Japan Waterworks Law, the fluoride concentration of tap water should be kept at a level not exceeding $0.8 \mathrm{ppm}$. However, in fact, the concentration of fluoride in the public water supply in most districts of Japan has been reported to be less than $0.1 \mathrm{ppm}^{1,2)}$. The World Health Organization (WHO) recommends a concentration of $0.5-1.0 \mathrm{ppm}$ of fluoride in the drinking water for

Received on October 14, 2005

Accepted on January 12, 2006 the prevention of dental caries ${ }^{3)}$.

Many kinds of fluoride-containing toothpaste are available throughout the world. In Japan, 87\% of the toothpastes currently on the market contain some form of fluoride ${ }^{4}$. It has been reported that preventive fraction of fluoride-containing toothpastes for the dental caries is $20-40 \%{ }^{5}$. Moreover, it has been suggested that fluoride-containing mouthrinses may also have an anti-caries effect in children ${ }^{6}$, and the daily use of mouthrinses containing $0.2 \%$ sodium fluoride has been shown to effectively reduce the incidence of dental caries ${ }^{7}$. In Japan, two mouthrinses containing fluoride are classified as dangerous drugs and supplied only in a powdered state. Because many mouthrinses contained fluoride are available in Europe, we carried out a field survey to gain an understanding of the market 
Table 1a The mouthrinses that contained fluoride in Athens, Greece

\begin{tabular}{|c|c|c|c|c|}
\hline Campany & Product name & $\begin{array}{l}\text { Country of } \\
\text { production }\end{array}$ & Ingredient & $\begin{array}{c}\text { Fluoride } \\
\text { Concentration }\end{array}$ \\
\hline \multirow{2}{*}{$\begin{array}{l}\text { COLGATE- } \\
\text { PALMOLIVE }\end{array}$} & $\begin{array}{c}\text { Plax } \\
\text { Soft Minto }\end{array}$ & - & $\begin{array}{c}\text { Aqua, Glycerin, Alcohol, Propylene Glycol, Sorbitol, PEG-40 } \\
\text { Sorbitan Diisostearate, Sodium Benzoate, Menthol, Aroma, } \\
\text { Cetylpyridium Chloride, Sodium Fluoride, Sodium Saccharin, } \\
\text { CI } 42090, \text { CI } 47005\end{array}$ & $0.025 \%$ \\
\hline & $\begin{array}{c}\text { Plax } \\
\text { Original }\end{array}$ & - & $\begin{array}{c}\text { Aqua, Sorbitol, Alcohol, Glycerin, Sodium Lauryl Sulfate, Sodium } \\
\text { Methyl Cocoyl Taurate, PVM/MA Copolymer, Aroma, Menthol, } \\
\text { Sodium Hydroxide, Disodium Phosphate, Tricrosan, Sodium Fluoride, } \\
\text { Sodium Saccharin, CI } 16035\end{array}$ & $0.025 \%$ \\
\hline Guaber & BLAN X & Italy & $\begin{array}{l}\text { Aqua, Glycerin, Sorbitol, Polysorbate 20, Cetylpyridium Chloride, } \\
\text { Usnea Barbata, Plantago Major, Sodium Bicarbonate, Mentha } \\
\text { Piperita, Anethole, Menthol, Allantoin, Propylene Glycol, Disodium } \\
\text { Phosphate, Sodium Lauryl Sulfate, Sodium Benzoate, Sodium } \\
\text { Saccharin, Sodium Fluoride, Imidazolidinyl Urea, Citric Acid, } \\
\text { Terasodium EDTA, Eugenol, CI 42051, CI } 19140\end{array}$ & $0.05 \%$ \\
\hline Johnson and Johnson & $\begin{array}{c}\text { ACT } \\
\text { FLUORIDE } \\
\text { Classical Cinnamon }\end{array}$ & - & $\begin{array}{l}\text { Aqua, Glycerin, Propylene Glycol, Poloxamer 407, Polysorbate } 80, \\
\text { Aroma, Limonene, Sodium Benzoate, Sodium Fluoride, Sodium } \\
\text { Phosphate, Disodium Phosphate, Benzyl Alcohol, Cetylpyridium } \\
\text { Chloride, Sodium Saccharin, Calcium Disodium EDTA, CI } 17200\end{array}$ & - \\
\hline Johnson and Johnson & $\begin{array}{l}\text { ACT } \\
\text { FLUORIDE } \\
\text { Fresh Mint }\end{array}$ & - & $\begin{array}{c}\text { Aqua, Sorbitol, Alcohol, Poloxamer 407, Sodium Benzoate, Sodium } \\
\text { Fluoride, Polysorbate 80, Sodium Phosphate, Sodium Saccharin, } \\
\text { Calcium Disodium EDTA, Aroma, Cinnamal, Limonene, CI 42090, } \\
\text { CI } 18965\end{array}$ & - \\
\hline Pfizer & LISTERINE & - & $\begin{array}{l}\text { Aqua, Alcohol, Sorbitol, Aroma, Poloxamer 407, Benzoic acid, } \\
\text { Sodium Saccharin, Eucalyptol, Methyl salicylate, Thymol, Menthol, } \\
\text { Sodium Benzoate, Sodium Fluoride, CI 42053, CI } 47005\end{array}$ & $100 \mathrm{ppm}$ \\
\hline \multirow[t]{2}{*}{ GABA } & elmex & - & $\begin{array}{l}\text { Aqua, Glycerin, Propylene Glycol, PEG-40 Hydrogenated Castor oil, } \\
\text { Olaflur, Aroma, PVP/Dimethylaminoethylmethacrylate Polycarbamyl } \\
\text { Polyglycol Ester, Saccharin, Hydroxyethylcellulose, Potassium } \\
\text { Fluoride, Potassium Hydroxide, Polyaminopropyl Biguanide }\end{array}$ & $250 \mathrm{ppm}$ \\
\hline & $\begin{array}{c}\text { elmex } \\
\text { anti-caries }\end{array}$ & - & $\begin{array}{c}\text { Aqua, PEG-40 Hydrogenated Castor oil, Olaflur, Aroma, Potassium } \\
\text { Acesulfame, Sodium Fluoride, Polyaminopropyl Biguanide, } \\
\text { Hydrochroric Acid }\end{array}$ & $250 \mathrm{ppm}$ \\
\hline Glaxo Smith Kline & SENSODYNE & - & $\begin{array}{l}\text { Aqua, Alcohol, Sorbitol, Poloxamer 407, Potassium Chloride, } \\
\text { Methylparaben, Aroma, Sodium Saccharin, Sodium Benzoate, } \\
\text { Propylparaben, Sodium Fluoride, Triclosan, CI } 42051\end{array}$ & - \\
\hline INTERMED & $\begin{array}{c}\text { Medinol } \\
\text { MOUTHWASH }\end{array}$ & Greece & $\begin{array}{c}\text { Aqua, Sorbitol, Xylitol, PEG-40 Hydrogenated Castor oil, PVP, } \\
\text { Poloxamer 407, Olaflur, Propylene Glycol, Stannous Fluoride, } \\
\text { Sodium Fluoride, Zinc Gluconate, Ammonium Glycyrrhizate, } \\
\text { Cetylpridinium Chloride, Panthenol, Bisabolol, Allantoin, } \\
\text { Sodium Saccharin, Aroma, CI } 42090\end{array}$ & - \\
\hline CICCARELLI & CAPITANO & Italy & $\begin{array}{c}\text { Aqua, Propylene Glycol, PEG-40 Hydrogenated Castor oil, } \\
\text { Sodium Benzoate, Sodium Lauryl Sulfate, Polysorbate 20, Aroma, } \\
\text { Lactic Acid, Sodium Lactate, Sodium Bicarbonate, Sodium Fluoride, } \\
\text { Glycerin, Cetylpyridium Chloride, Centella Asiatica, } \\
\text { 2-bromo-2-nitropropane-1,3-diol, Triclosan, Sodium Saccharin, } \\
\text { CI } 42051\end{array}$ & - \\
\hline
\end{tabular}

— indicates the undocumented country of production or concentration of fluoride.

sales of these products in three countries. We visited pharmacies, supermarkets, general shops, and kiosks in Athens, Amsterdam, and Geneva, and purchased any mouthrinses that contained fluoride, according to the ingredients label. The products obtained are shown in Table 1a-c. A total of 11, 14, and 17 fluoride-containing mouthrinses were obtained in Athens, Amsterdam, and Geneva, respectively. The concentration of fluoride in these products ranged from $100 \mathrm{ppm}$ to $500 \mathrm{ppm}$.

Among the 42 products purchased in this study, 22 contained the Alcohol and 13 contained the Cetylpyridium Chloride, these ingredients may be effects as anti-microbe drugs. Thirty-five products contained Sodium Saccharin and 23 contained Sorbitol as edulcorant. There are not clear differences in the ingredients between three countries, however, some products that contained the Zinc Chloride 
Table 1b The mouthrinses that contained fluoride in Amsterdam, the Netherlands

\begin{tabular}{|c|c|c|c|c|}
\hline Campany & Product name & $\begin{array}{l}\text { Country of } \\
\text { production }\end{array}$ & Ingredient & $\begin{array}{c}\text { Fluoride } \\
\text { Concentration } \\
\end{array}$ \\
\hline \multirow{3}{*}{ DA } & $\begin{array}{l}\text { Mondwater } \\
\text { total care }\end{array}$ & - & $\begin{array}{l}\text { Aqua, Alcohol, Sorbitol, Polysorbate 20, Aroma, Allantoin, } \\
\text { Cethylpyridinium Chloride, Sodium Fluoride, Sodium Saccharin, } \\
\text { Sodium Benzoate, Menthol, Zinc Chloride, Limonene, CI } 42051\end{array}$ & - \\
\hline & $\begin{array}{c}\text { Mondwater } \\
\text { sensitive and whitening }\end{array}$ & - & $\begin{array}{c}\text { Aqua, Sorbitol, Potassium Citrate, Polysorbate 20, Pentasodium } \\
\text { Triphosphate, Lactic Acid, Aroma, Sodium Fluoride, } \\
\text { Cethylpyridinium Chloride, Sodium Saccharin, Sodium Benzoate, } \\
\text { Sodium Methylparaben, Caramel }\end{array}$ & - \\
\hline & $\begin{array}{c}\text { Mondwater } \\
\text { extra sterk and fris }\end{array}$ & - & $\begin{array}{c}\text { Aqua, Alcohol, Sorbitol, Polysorbate 20, Aroma, Cethylpyridinium } \\
\text { Chloride, Sodium Fluoride, Sodium Saccharin, Sodium Benzoate, } \\
\text { CI } 18965, \text { CI } 42051\end{array}$ & - \\
\hline Pfizer & LISTERINE & - & $\begin{array}{l}\text { Aqua, Alcohol, Sorbitol, Aroma, Poloxamer 407, Benzoic acid, } \\
\text { Sodium Saccharin, Eucalyptol, Methyl salicylate, Thymol, Menthol, } \\
\text { Sodium Benzoate, Sodium Fluoride, CI 42053, CI } 47005\end{array}$ & $100 \mathrm{ppm}$ \\
\hline \multirow[t]{2}{*}{ GABA } & elmex & - & $\begin{array}{l}\text { Aqua, Glycerin, Propylene Glycol, PEG-40 Hydrogenated Castor oil, } \\
\text { Olaflur, Aroma, PVP/Dimethylaminoethylmethacrylate Polycarbamyl } \\
\text { Polyglycol Ester, Saccharin, Hydroxyethylcellulose, Potassium } \\
\text { Fluoride, Potassium Hydroxide, Polyaminopropyl Biguanide }\end{array}$ & $250 \mathrm{ppm}$ \\
\hline & $\begin{array}{l}\text { elmex } \\
\text { anti-caries }\end{array}$ & - & $\begin{array}{c}\text { Aqua, PEG-40 Hydrogenated Castor oil, Olaflur, Aroma, Potassium } \\
\text { Acesulfame, Sodium Fluoride, Polyaminopropyl Biguanide, } \\
\text { Hydrochloric Acid }\end{array}$ & $250 \mathrm{ppm}$ \\
\hline \multirow{3}{*}{ Glaxo Smith Kline } & SENSODYNE & - & $\begin{array}{l}\text { Aqua, Alcohol, Sorbitol, Poloxamer 407, Potassium Chloride, } \\
\text { Sodium Fluoride, Triclosan, Sodium Saccharin, Sodium Benzoate, } \\
\text { Methylparaben, Polyparaben, Aroma, CI } 42051\end{array}$ & - \\
\hline & $\begin{array}{l}\text { Odol med } \\
\text { Anti-plaque }\end{array}$ & - & $\begin{array}{c}\text { Aqua, Alcohol, Sorbitol, Sodium Benzoate, Poloxamer 338, } \\
\text { Cetylpyridium Chloride, PEG-60 Hydrogenated Castor oil, } \\
\text { Sodium Fluoride, Aroma, Sodium Saccharin, Citric Acid, CI } 42053\end{array}$ & - \\
\hline & $\begin{array}{l}\text { Odol med } \\
\text { green }\end{array}$ & - & $\begin{array}{l}\text { Aqua, Alcohol, Flavour, Cocamidoproply Betaine, Sodium Saccharin, } \\
\text { Cetylpyridium Chloride, Sodium Fluoride, PEG-60 Hydrogenated } \\
\text { Castor oil, Sodium Benzoate, CI 42051, CI 75810, CI } 15985\end{array}$ & - \\
\hline Ardoz Research & $\begin{array}{c}\text { Vitint } \\
\text { Mond-Conditioner }\end{array}$ & Netherland & $\begin{array}{l}\text { Aqua, Glycerin, Sodium Lauryl Sulfate, PEG-40 Hydrogenated Castor } \\
\text { oil, Sodium Gluconate, Cellulose Gum, Aroma, Sodium Citrate, } \\
\text { Sodium Methylparaben, Sodium Perborate, Citric Acid, Sodium } \\
\text { Chloride, Sodium Fluoride, Magnesium Sulfate, Sodium Saccharin }\end{array}$ & $226 \mathrm{ppm}$ \\
\hline Purity Laboratories & $\begin{array}{c}\text { Natural white } \\
\text { whitening mouthrinse }\end{array}$ & - & $\begin{array}{c}\text { Aqua, Alcohol, Glycerin, PEG-40 Hydrogenated Castor oil, Sodium } \\
\text { Lauryl Sulfate, Triclosan, Sodium Saccharin, Sodium Benzoate, } \\
\text { Aroma, Sodium Fluoride, CI } 42090\end{array}$ & $0.05 \%$ \\
\hline Oral B & $\begin{array}{l}\text { Tanden en Tandvlees } \\
\text { Dents et Gencivlees }\end{array}$ & UK & $\begin{array}{l}\text { Aqua, Glycerin, Polysorbate 20, Aroma, Methylparaben, } \\
\text { Cetylpyridium Chloride, Sodium Fluoride, Sodium Saccharin, } \\
\text { Sodium Benzoate, Propylparaben, CI 42051, CI } 47005\end{array}$ & $0.05 \%$ \\
\hline Albert Heijn & $\begin{array}{l}\text { Mond } \\
\text { Water }\end{array}$ & Netherland & $\begin{array}{c}\text { Aqua, Glycerin, Cetylpyridium Chloride, Aroma, Sodium Fluoride, } \\
\text { Cinnamal, Limonene, PEG-40 Hydrogenated Castor oil, Sodium } \\
\text { Benzoate, 2-bromo-2-nitropropane-1,3-diol, Sodium Saccharin, } \\
\text { Citric acid, Eugenol, CI } 42051\end{array}$ & - \\
\hline etos & $\begin{array}{l}\text { MONDWATER } \\
\text { ANTI-SEPTISCH }\end{array}$ & Netherland & $\begin{array}{c}\text { Aqua, Alcohol, Xylitol, PEG-40 Hydrogenated Castor oil, Sodium } \\
\text { Benzoate, Eucalyptus Globulus, Menthol, Sodium Fluoride, } \\
\text { Methyl salicylate, Thymus Vulgaris, Potassium Sorbate, Parfum, } \\
\text { Cinnamal, D-limonene, Eugenol, CI } 19140\end{array}$ & $0.04 \%$ \\
\hline
\end{tabular}

— indicates the undocumented country of production or concentration of fluoride.

for the prevention of the malodors are found in Switzerland.

In Europe, water fluoridation has not been universally implemented, and fluoride is provided via mouthrinses and toothpastes. In 2000, the DMF index of caries prevalence was 2.2 in Greece, 0.6 in the Netherlands, and 0.9 in Switzerland. In all three countries, the DMF index was lower than the 2.4 reported for Japan in $1999^{8)}$. It has reported topical fluorides (varnishes, gels and mouthrinses) used in addition to fluoride toothpaste achieve a modest reduction in caries compared to toothpaste used alone ${ }^{9)}$, and intensive fluoridation is effective in preventing enamel and dentine caries ${ }^{10)}$. One of the 
Table 1c The mouthrinses that contained fluoride in Geneva, Switzerland

\begin{tabular}{|c|c|c|c|c|}
\hline Campany & Product name & $\begin{array}{l}\text { Country of } \\
\text { production }\end{array}$ & Ingredient & $\begin{array}{c}\text { Fluoride } \\
\text { Concentration }\end{array}$ \\
\hline \multirow{5}{*}{ MIGROS } & $\begin{array}{l}\text { CANDIDA MULTICARE } \\
\text { DENTAL FLUID }\end{array}$ & \multirow{7}{*}{ Switzerland } & $\begin{array}{c}\text { Aqua, Glycerin, Sorbitol, Glycine, PEG-60 Hydrogenated Castor oil, } \\
\text { Aroma, Zinc Chloride, Sodium Fluoride, Sodium Saccharin, } \\
\text { Domiphen Bromide, Cetylpyridinum Chloride }\end{array}$ & \multirow{5}{*}{$220 \mathrm{mg} / \mathrm{kg}$} \\
\hline & $\begin{array}{l}\text { CANDIDA SENSITIVE } \\
\text { PLUS }^{+} \\
\text {DENTAL FLUID }\end{array}$ & & $\begin{array}{l}\text { Aqua, Glycerin, Sorbitol, Poloxamer 407, PEG-60 Hydrogenated } \\
\text { Castor oil, Potassium Citrate, Aroma, Methylparaben, Panthenol, } \\
\text { Sodium Saccharin, Zinc Chloride, Sodium Fluoride, Cetylpyridium } \\
\text { Chloride, Citric Acid, BHT, Eugenol, Limonene }\end{array}$ & \\
\hline & $\begin{array}{l}\text { CANDIDA ANTI } \\
\text { TARTARO } \\
\text { DENTAL FLUID }\end{array}$ & & $\begin{array}{l}\text { Aqua, Alcohol, Glycerin, Sorbitol, Tetrasodium Pyrophosphate, } \\
\text { Disodium Pyrophosphate, PEG-60 Hydrogenated Castor oil, } \\
\text { Methylparaben, Aroma, Sodium Fluoride, Sodium Saccharin }\end{array}$ & \\
\hline & $\begin{array}{l}\text { CANDIDA ACTIVE } \\
\text { FRESH DENTAL } \\
\text { FLUID }\end{array}$ & & $\begin{array}{c}\text { Aqua, Glycerin, Sorbitol, Glycine, PEG-60 Hydrogenated Castor oil, } \\
\text { Aroma, Zinc Chloride, Sodium Fluoride, Sodium Saccharin, } \\
\text { Domiphen Bromide, Cetylpyridinum Chloride, } \\
\text { Polyaminopropyl Biguanide }\end{array}$ & \\
\hline & $\begin{array}{l}\text { CANDIDA PARODIN } \\
\text { DENTAL FLUID }\end{array}$ & & $\begin{array}{c}\text { Aqua, Glycerin, Sorbitol, Poloxamer 407, PEG-60 Hydrogenated } \\
\text { Castor oil, Glycyrrhiza Glabra, Propylene Glycol, Allantoin, Aroma, } \\
\text { Panthenol, Sodium Saccharin, Sodium Fluoride, Bisabolol, } \\
\text { Polyaminopropyl Biguanide, BHT }\end{array}$ & \\
\hline \multirow{3}{*}{ GABA } & Meridol & & $\begin{array}{l}\text { Aqua, Xylitol, PVP, PEG-40 Hydrogenated Castor oil, Olaflur, } \\
\text { Aroma, Stannous Fluoride, Sodium Saccharin, CI } 42051\end{array}$ & $0.025 \%$ \\
\hline & $\begin{array}{l}\text { elmex } \\
\text { sensitive }\end{array}$ & & $\begin{array}{l}\text { Aqua, Glycerin, Propylene Glycol, PEG-40 Hydrogenated Castor oil, } \\
\text { Olaflur, Aroma, PVP/Dimethylaminoethylmethacrylate Polycarbamyl } \\
\text { Polyglycol Ester, Saccharin, Hydroxyethylcellulose, Potassium } \\
\text { Fluoride, Potassium Hydroxide, Polyaminopropyl Biguanide }\end{array}$ & $250 \mathrm{ppm}$ \\
\hline & $\begin{array}{l}\text { elmex } \\
\text { anti-caries }\end{array}$ & - & $\begin{array}{c}\text { Aqua, PEG-40 Hydrogenated Castor oil, Olaflur, Aroma, Potassium } \\
\text { Acesulfame, Sodium Fluoride, Polyaminopropyl Biguanide, } \\
\text { Hydrochloric Acid }\end{array}$ & $250 \mathrm{ppm}$ \\
\hline \multirow{2}{*}{$\begin{array}{l}\text { COLGATE- } \\
\text { PALMOLIVE }\end{array}$} & Colgate Rinc Dent Sans & - & $\begin{array}{c}\text { Aqua, Glycerin, Propylene Glycol, Sorbitol, Sodium Phosphate, } \\
\text { Poloxamer 407, Sodium Benzoate, Disodium Phosphate, Aroma, } \\
\text { Cetylpyridium Chloride, Sodium Saccharin, Sodium Fluoride, } \\
\text { Cinnamal }\end{array}$ & $0.025 \%$ \\
\hline & Colgate Total & - & $\begin{array}{l}\text { Aqua, Sorbitol, Alcohol, Glycerin, Aroma, Disodium Phosphate, } \\
\text { PVM/MA Copolymer, Sodium Fluoride, Sodium Hydroxide, Sodium } \\
\text { Sulfate, Sodium Methyl Cocoyl Taurate, Sodium Saccharin, Tricosan }\end{array}$ & - \\
\hline Glaxo Smith Kline & Odol-dent 3 MINT & - & $\begin{array}{c}\text { Aqua, Alcohol, Sodium Benzoate, Poloxamer 338, Cetylpyridium } \\
\text { Chloride, PEG-60 Hydrogenated Castor oil, Sodium Fluoride, Aroma, } \\
\text { Sodium Saccharin, Citric Acid }\end{array}$ & - \\
\hline LEVER FABERGE & Mentadent & - & $\begin{array}{l}\text { Aqua, Alcohol, Sorbitol, Sodium Fluoride, Zinc Sulfate, Glycine, } \\
\text { Sodium Lauryl Sulfate, Aroma, Poloxamer 188, Sodium Saccharin, } \\
\text { CI 16255, CI 42051, CI 47005 }\end{array}$ & $225 \mathrm{ppm}$ \\
\hline Johnson and Johnson & $\begin{array}{l}\text { ACT } \\
\text { Dental Fluid junior }\end{array}$ & - & $\begin{array}{l}\text { Aqua, Glycerin, Propylene Glycol, Poloxamer 407, Polysorbate 80, } \\
\text { Aroma, Sodium Benzoate, Sodium Fluoride, Sodium Phosphate, } \\
\text { Disodium Phosphate, Benzyl Alcohol, Cetylpyridium Chloride, } \\
\text { Sodium Saccharin, Calcium Disodium EDTA, CI } 17200\end{array}$ & - \\
\hline Johnson and Johnson & $\begin{array}{l}\text { ACT } \\
\text { FLUORIDE } \\
\text { Classical Cinnamon }\end{array}$ & - & $\begin{array}{l}\text { Aqua, Glycerin, Propylene Glycol, Poloxamer 407, Polysorbate 80, } \\
\text { Aroma, Limonene, Sodium Benzoate, Sodium Fluoride, Sodium } \\
\text { Phosphate, Disodium Phosphate, Benzyl Alcohol, Cetylpyridium } \\
\text { Chloride, Sodium Saccharin, Calcium Disodium EDTA, CI } 17200\end{array}$ & - \\
\hline Johnson and Johnson & $\begin{array}{l}\text { ACT } \\
\text { FLUORIDE } \\
\text { Fresh Mint }\end{array}$ & - & $\begin{array}{l}\text { Aqua, Sorbitol, Alcohol, Poloxamer 407, Sodium Benzoate, Sodium } \\
\text { Fluoride, Polysorbate 80, Sodium Phosphate, Sodium Saccharin, } \\
\text { Calcium Disodium EDTA, Aroma, Cinnamal, Limonene, CI 42090, } \\
\text { CI } 18965\end{array}$ & - \\
\hline Dr. Wild and Co. & $\begin{array}{l}\text { EMOFORM } \\
\text { with Fluor }\end{array}$ & Switzerland & $\begin{array}{c}\text { Aqua, Propylene Glycol, Potassium Chloride, Sodium Laureth } \\
\text { Sulfate, Aroma, Sodium Fluoride, Sodium Chloride, } \\
\text { Sodium Saccharin }\end{array}$ & $250 \mathrm{ppm}$ \\
\hline Pfizer & $\begin{array}{l}\text { LISTERINE } \\
\text { ANTI-BACTERIAL } \\
\text { MONDWATER }\end{array}$ & - & $\begin{array}{l}\text { Aqua, Alcohol, Sorbitol, Aroma, Poloxamer 407, Benzoic acid, } \\
\text { Sodium Saccharin, Eucalyptol, Methyl salicylate, Thymol, Menthol, } \\
\text { Sodium Benzoate, Sodium Fluoride, CI 42053, CI } 47005\end{array}$ & $100 \mathrm{ppm}$ \\
\hline
\end{tabular}

— indicates the undocumented country of production or concentration of fluoride. 
reasons of this difference may be derived from the easily-obtainable fluoride-containing mouthrinses as commercialized products in Europe, but not in Japan.

In conclusion, the availability of fluoridecontaining mouthrinses may be determined and influenced by local policy; however, there is little doubt that these products contribute to the prevention of dental caries.

\section{References}

1) Takeuchi, K., Nakagaki, H., Toyama, Y., Kimata, N., Ito, F., Robinson, C., Weatherell, J.A., St-osser, L. and Kunzel, W.: Fluoride concentrations and distribution in premolars of children from low and optimal fluoride areas. Caries Res 30: 76-82, 1996.

2) Mihata, T., Toda, S., Komiyama, M., Kushida, M., Song, W., Arajawa, H., Uchimura, N. and lizuka, Y.: Study on the fluoride concentration in drinking water in Japan-Results around Kanto area-. J Dent Health 49: 294-303, 1999.

3) Fluorides and oral health: Report of a WHO expert committee on oral health status and fluoride use. World Health Organization, Geneva, 1994.

4) Life of Fluoride Products of Japan. Koko Hoken Kyoukai, Tokyo, 2004.
5) Stookey, G.K., DePaola, P.F., Featherstone, J.D., Fejerskov, O., Moller, I.J., Rotberg, S., Stephen, K.W. and Wefel, J.S.: A critical review of the relative anticaries efficacy of sodium fluoride and sodium monofluorophosphate dentifrices. Caries Res 27: 337-360, 1993.

6) Twetman, S., Petersson, L., Axelsson, S., Dahlgren, H., Holm, A.K., Kallestal, C., Lagerlof, F., Lingstrom, P., Mejare, I., Nordenram, G., Norlund, A. and Soder, B.: Caries-preventive effect of sodium fluoride mouthrinses: a systematic review of controlled clinical trials. Acta Odontol Scand 62: 223-230, 2004.

7) Wyatt, C.C.L. and MacEntee, M.I.: Caries management for institutionalized elders using fluoride and chlorhexidine mouthrinses. Community Dent Oral Epidemiol 32: 322-328, 2004.

8) Caries for 12-Year-Olds by Country/Area WHO Region, WHO, Language Recommendations. http: www.8020zaidan.or.jp/international/index.html, access day 2005.9.22.

9) Monopoli, M.: Topical fluorides provide additional benefit when used with fluoride toothpaste. Evid Based Dent 5: 38, 2004.

10) Ganss, C., Klimek, J., Brune, V. and Schurmann, A.: Effects of two fluoridation measures on erosion progression in human enamel and dentine in situ. Caries Res 38: 561-566, 2004. 